Corrección al abstract del artículo: Parechovirus como agente etiológico de meningitis y/o sepsis viral en lactantes, de Valentina Gutiérrez y cols., Rev Chilena Infectol 2016; 4: 380-8

\title{
Debe decir:
}

Introduction: Human parechovirus (HPeV) belongs to the Picornaviridae family and has been described in viral meningoencephalitis ans sepsis like illness in infants. Until now, 16 genotypes have been recognized, the most common are HPeV 1, 2 and 3; type 3 is most severe. Aims: To estimate the frequency of HPeV etiology in viral meningoencephalitis and sepsis in infants and characterize clinical and molecular aspects of infection. Methods: Between October 2013 and March 2015 we collected CSF samples, plasma, nasopharyngeal swabs and/or stools of patients younger than two years with suspected sepsis and/or viral meningitis. Samples were obtained from laboratory storage sites and from hospitalized patients. HPeV was diagnosed by real-time polymerase chain reaction (PCR) assay against the 5'UTR region. Positive samples were genotyped by sequencing a 304pb segment in VP3/VP1 overlapping region obtained with a nested PCR. Results: Overall $\mathrm{HPeV}$ detection rate was 18,6\% (11/59 patients), distributed in $8.7 \%(4 / 46)$ laboratory's samples and 53.8\% (7/13) of samples from hospitalized patients; mean age was 49 days (18 days-6 months). Most common clinical signs (11/11 patients) were irritability, inappetance, and fever (magnitude $38-38.8^{\circ} \mathrm{C}$ ). All six samples genotyped were HPeV 3. Conclusions: HPeV should be considered as a relatively significant etiologic agent of viral meningoencephalitis and sepsis in infants. 